allele, was detected in 12 heterozygous patients with. The p.Q141K variant, detected in the homozygous stage in three patients and in 31 patients as heterozygous variant. Heterozygous rare variants p.R45Q, p. G354R, p.A607V, and novel variant p.E344D were detected in one heterozygous patient each; heterozygous $\mathrm{p} . \mathrm{M} 131 \mathrm{I}$ and $\mathrm{p} . \mathrm{R} 236 \mathrm{X}$ in two patients.

Conclusion: In this study, significantly higher frequency of dysfunctional ABCG2 variants, common and rare, in comparison with common European population, were identified. On the other hand, the frequency of probably protective allele variant $p . V 12 \mathrm{M}$ was significantly lower in CKD cohort (MAF in our cohort $=0,036$ / $M A F$ in the European population $=0,061$ ). Further analysis of $A B C G 2$ association with $C K D$ events via $A B C G 2$ inflammation role is necessary. In conclusions, our finding of one novel, five rare and two common non-synonymous ABCG2 allelic variants in a sample of 167 CKD patients suggests that the ABCG2 variants should be considered a risk factor for CKD.

REFERENCES:

[1] Toyoda $Y$, et al. Functional Characterization of Clinically-Relevant Rare Variants in ABCG2 Identified in a Gout and Hyperuricemia Cohort. Cells. $2019 ; 18 ; 8(4)$

Acknowledgements: This study was supported by the grant from the Czech Republic Ministry of Health RVO 00023728

Disclosure of Interests: None declared

DOI: 10.1136/annrheumdis-2021-eular.2084

\section{POS0355 1 ASSOCIATIONS BETWEEN HLA-DRB1 SHARED EPITOPES ALLELES AND ANTI-RA33 ANTIBODIES IN DIFFERENT SUBSETS OF RHEUMATOID ARTHRITIS IN MALAYSIAN POPULATION}

A. F. Nurul-Aain ${ }^{1}$, S. S. Ch'ng ${ }^{2}$, H. Baharuddin ${ }^{2,3}$, M. Mohd Zain², I. S. Lau ${ }^{2}$ H. Mohd Yusoof ${ }^{2}$, A. Ahmad ${ }^{2}$, A. Rosman ${ }^{2}$, A. A. Siti-Aisyah ${ }^{1}$, L. Padyukov ${ }^{4}$ L. Alfredsson ${ }^{5}$, L. Klareskog ${ }^{4}$, J. Rönnelid ${ }^{6}$, C. L. Too ${ }^{1,4}$ on behalf of the MyEIRA Study Group. ${ }^{1}$ Allergy and Immunology Research Centre, Institute for Medical Research, Ministry of Health Malaysia, Selangor, Malaysia; ${ }^{2}$ Rheumatology Unit, Department of Internal Medicine, Selayang Hospital, Ministry of Health Malaysia, Selangor, Malaysia; ${ }^{3}$ Medical Faculty, Hospital Selayang Campus, University Institute Technology Mara (UiTM), Selangor, Malaysia; ${ }^{4}$ Division of Rheumatology, Department of Medicine, Karolinska Institutet and Karolinska University Hospital, Stockholm, Sweden; ${ }^{5}$ Institute of Environmental Medicine, Karolinska Institutet, Stockholm, Sweden; ${ }^{6}$ Department of Immunology, Genetics and Pathology, Uppsala University, Uppsala, Sweden

Background: The mechanisms affecting anti-RA33 antibody's involvement in RA pathogenesis is still unclear. Refining our understanding of anti-RA33's role in RA in relation to known RA-associated genes and serological elements is needed.

Objectives: We investigated the relationship between RA-associated HLADRB1 epitope (SE) allele and presence of anti-RA33 antibodies in different serological subsets of rheumatoid arthritis in a Malaysian population.

Methods: Serum samples from 550 RA cases comprising seronegative (negative for anti-CCP2, IgG and $\lg M, n=250$ ), seropositive (triple-autoantibody positive, $n=150)$, singular anti-CCP2 positive $(n=100)$, and double RF positive RA $(n=50)$ were chosen from the Malaysian Epidemiologica Investigations of RA (MyEIRA) case-control study. Three hundred MyEIRA population controls were used for comparison. All serum samples were assayed using a commercial anti-RA33 ELISA kit. All genetic samples were genotyped for four-digit HLA-DRB1 alleles using the PCR-SSO method on Luminex platform.

Results: The proportions of anti-RA33 positive was $20.9 \%$ in all RA cases (i.e. $34 \%$ in RF only positive RA; $25 \%$ in seropositive RA; $18 \%$ in seronegative RA and $18 \%$ in anti-CCP2 only positive RA). The HLA-DRB1 shared epitope alleles were significantly associated with anti-RA33 positive in the seropositive RA subgroup $(\mathrm{OR}=6.9,95 \% \mathrm{Cl} 1.4-34.8 ; \mathrm{p}=0.02)$. We observed significant association between anti-RA33 negative and HLA-DRB1 SE alleles among the seropositive RA patients $(\mathrm{OR}=4.5,95 \% \mathrm{Cl} 2.8-7.2 ; \mathrm{p}<0.001)$ and among $\mathrm{CCP}$ only positive RA (OR=4.4; 95\% Cl 2.6-7.4; $p<0.01)$. No association was observed between anti-RA33 status and HLA-DRB1 SE alleles in seronegative RA and RF only positive RA.

Conclusion: The HLA-DRB1 SE alleles increased the risk of seropositive and CCP only positive RA independent of anti-RA33 positivity. REFERENCES:

[1] Boeters, Debbie M et al. "The 2010 ACR/EULAR criteria are not sufficiently accurate in the early identification of autoantibody-negative rheumatoid arthritis: Results from the Leiden-EAC and ESPOIR cohorts." Seminars in arthritis and rheumatism vol. 47,2 (2017): 170-174.

[2] de Brito Rocha, Sara et al. "Clinical and pathophysiologic relevance of autoantibodies in rheumatoid arthritis." Advances in rheumatology (London, England) vol. 59,1 2. 17 Jan. 2019
Acknowledgements: The authors would like to thank the Director General of Health, Ministry of Health Malaysia for supporting this study. The authors are also indebted to participants for their kind participation. This study was financially supported by the Ministry of Health, Malaysia (JPP-IMR 08-012).

Disclosure of Interests: None declared

DOI: 10.1136/annrheumdis-2021-eular.2298

\section{POS0356 \\ THE RELATIONSHIP OF GENETICS AND CLINICALLY SUSPECT ARTHRALGIA IN RA DEVELOPMENT ASSESSED USING HC, CSA AND RA PATIENTS}

M. Maurits ${ }^{1}$, F. Wouters ${ }^{1}$, E. Niemantsverdriet ${ }^{1}$, T. Huizinga ${ }^{1}$, A. van der Helm - van Mil ${ }^{1}$, R. Knevel ${ }^{1} .{ }^{1}$ Leiden University Medical Center (LUMC), Rheumatology, Leiden, Netherlands

Background: The identification of a pre-RA stage of patients with clinically suspect arthralgia (CSA) has proven to be beneficial in the early detection of Rheumatoid disease. Similarly, genetic susceptibility studies have identified importan genetic risk factors for the development of (CCP positive) RA. ${ }^{1}$ The question that arises is whether these findings represent independent etiological pathways and could therefore be complimentary in the early diagnosis of RA.

Objectives: To corroborate the knowledge of genetic differences between $\mathrm{HC}$ and RA patients and extend it to include the CSA stage of disease.

Methods: We used three datasets sampled from the same region in the Netherlands: 1,085 healthy controls $(\mathrm{HC}), 530 \mathrm{CSA}$ and 1,277 RA patients. CSA patients were monitored for a median of 2 years for conversion into clinically apparent inflammatory arthritis (CSAc) or not (CSAnc) ${ }^{2}$ We assessed the association between HLA SE and disease stage using logistic regression. The analysis was repeated in the CCP positive and CCP negative strata of both the CSA and the RA populations.

Results: Consistent with previous studies, HLA SE was significantly enriched in RA patients compared to $\mathrm{HC}$ (OR 2.28) (Figure 1). HLA SE also differentiated HC vs CSAc (OR 1.69), CSAnc vs CSAc (OR 1.74), and CSAnc vs RA (OR 2.35). No difference was found in $\mathrm{HC}$ vs CSAnc and CSAc vs RA.

Conclusion: HLA SE is more prevalent in patients who developed (rheumatoid) arthritis than in both healthy controls and CSA patients who do not progress to arthritis. The results presented here seem to indicate a clear distinction between CSA patients who develop arthritis and those who do not. We therefore believe that known RA genetics play a role in the development of arthritis rather than the CSA symptoms. While this relationship varies by CCP status, an independen effect remains. Studies into the broader role of genetics beyond HLA SE are currently underway.

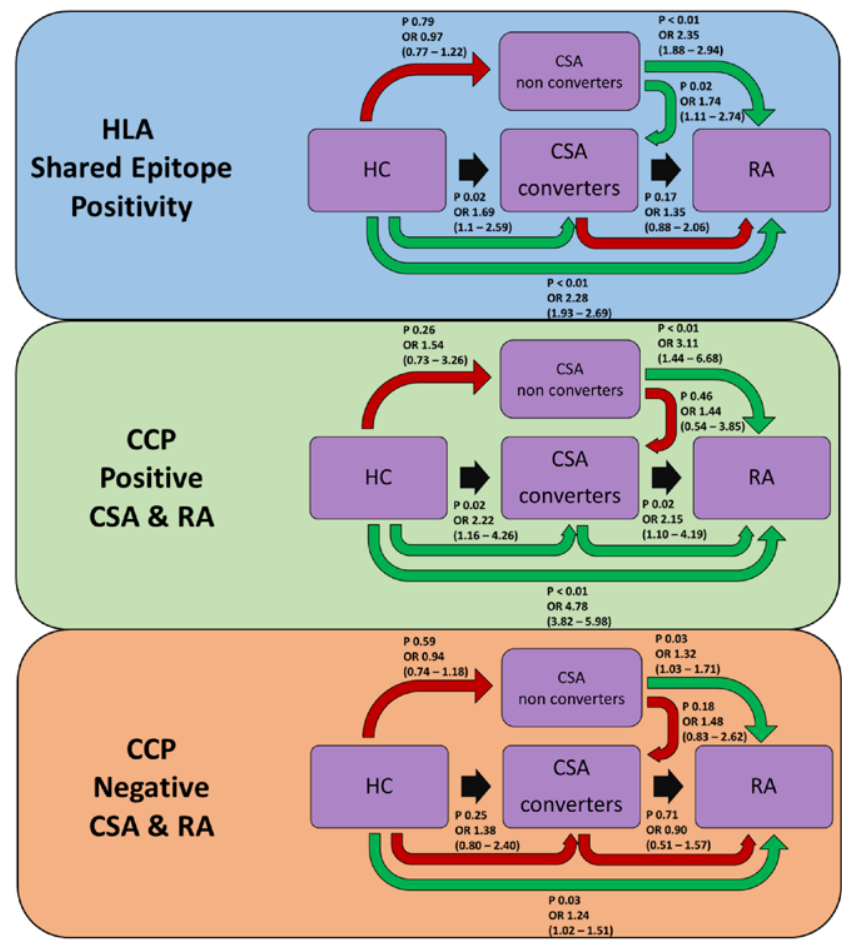

Figure 1. Distinguishing ability of HLA SE across HC, CSAc, CSAnc and RA in the full populations as well as in the CCP positive and negative stratifications. The arrowhead indicates the "case" status in each logistic regression. OR's $(95 \% \mathrm{Cl})$ derived from regression coefficients indicate the change in odds ratio attributable to HLA SE positivity. 